\title{
Preface
}

\section{Crossing over to the Future: Interdisciplinarity in Research and Higher Education}

\author{
MILENA ŽIC FUCHS \\ University of Zagreb, Croatia. Email: mzicfuch@ffzg.hr
}

On behalf of the Academia Europaea Organizing committee:

Chair: Milena Žic Fuchs

Erik De Corte, Leuven, Belgium, erik.decorte@ppw.kuleuven.be

Lars Engwall, Uppsala, Sweden, lars.engwall@fek.uu.se

Liesbet Geris, YAE, Liege, Belgium, liesbet.geris@ulg.ac.be

Svend Erik Larsen, Aarhus, Denmark, litsel@cc.au.dk

Bert Weckhuysen, Utrecht, The Netherlands, b.m.weckhuysen@uu.nl

In view of the increasing need for interdisciplinarity in order to handle present and future problems, the HERCulES Group within the Academia Europaea and the Wenner-Gren Foundations organized a conference entitled Crossing over to the Future: 'Interdisciplinarity' in Research and Higher Education, held from 18-20 May 2017 in Stockholm, Sweden. This conference was certainly not the first conference organized on the topic of 'interdisciplinarity', and certainly will not be the last. The reason for this lies in the fact that 'interdisciplinarity' is an ever-developing concept attempting to map a rapidly changing reality of knowledge production that covers not only research, but also higher education, and has great impact on the academic paths of early-career researchers. Thus, the three main sections of the conference covered three topics with the aim of addressing issues inherent in 'interdisciplinarity': (1) 'Interdisciplinarity' in research - obstacles and opportunities; (2) From research to teaching - principles and programmes, and (3) 'Interdisciplinarity' and the academic career path. Needless to say, the three topics mirror a complexity of relations and are definitely connected with science policies both at national and European levels as well as reflecting ever-present changes and the necessity of encompassing cultural 
concepts, both in the sense of 'cultures' inherent in disciplines as well as the wider concept of culture.

Even more importantly, the issues at hand were approached not only from theoretical perspectives but also from the views that come from concrete experiences and endeavours. Following these two fundamental perspectives, well-known actors in the academic community were invited to speak. Through their presentations as well as the discussions, old as well as new issues and views were identified and discussed. Thus, myriad questions surfaced, questions already present within the long history of 'interdisciplinarity', but also new points were brought up and ways forward were identified, such as: 'How to bridge gaps between disciplines and research domains?', 'What are the implications for resetting research agendas?', 'What are the implications for the global dimension?'. In addition, in relation to higher education, a further fundamental question was: "How to "reform" universities which (with a few exceptions) have not implemented systematic reforms of the traditional institutionalized boundaries between disciplines?'

Apart from institutional frameworks, whether in research or higher education, major questions such as to how do we disseminate 'knowledges' of all kinds to obtain future societal and natural sustainability, were interwoven throughout. More specifically: "How do we disseminate "knowledges" within academia but also to the general public?', and 'Can "interdisciplinarity" be the trigger for changing mind-sets in a constantly changing societal and natural environment?'

Much of the above, as already noted, has a history of different views and different interpretations as to how 'interdisciplinarity' is interwoven in research as well as university life. For example, more than 50 years have passed since C.P. Snow, in his famous Rede Lecture of $1959^{1}$ on the 'two cultures', stressed the duality of the natural sciences and the humanities as a seemingly self-evident reality organizing the academic production of knowledge. Snow argued that the university was a divided community with radically different cultures and languages. As is well known, his views have been greatly quoted, challenged and criticized. However, over the decades, the contemporary research landscape can no longer be mapped by simple dichotomies. Today, the concept of interdisciplinarity has slowly but surely come to the forefront of research interests and is seen as leading the way to answering major research questions, many of which are often known as the Grand Challenges confronting mankind today.

When one views what at present comes most frequently under the heading of the Grand Challenges (climate change, water resources, multiculturalism, migration, energy supplies, etc.), the two most dominant characteristics of this kind of research are encapsulated in the concepts of Inter/Multi/Transdisciplinarity and the Global Dimension, which in the propagated policy of the European Commission is called 'Open to the World'. The above stems from the global nature of the Grand Challenges themselves as well as from the fact that they do not easily fit within the traditional disciplinary boundaries. The complexity implied by interdisciplinary research challenges both research and higher education within all research domains. The complex nature of crossing boundaries and integrating different lines of thought brings to the forefront a reconceptualization of research itself as well as the education 
necessary for it. It is not possible to mention all the great thinkers that have stressed and developed the notion of 'interdisciplinarity' over the decades. However, it is necessary to mention the work of Jürgen Mittelstraß, ${ }^{2}$, who advocates the necessity of transforming 'empirical knowledge' into 'orientational knowledge', which is by definition culturally defined and socially implemented. Relevant is also the work of Nowotny, Scott and Gibbons ${ }^{3}$ on what is called Mode 2 knowledge production, that is, knowledge production seen as a process in which researchers collaborate in temporary networks to solve specific problems in the real world.

No matter whether 'interdisciplinarity' is primarily knowledge-led (research driven) or results from risk (crisis) induced research questions or is challenge-driven, the basic requirement for scholarly engagement across research domains is the capacity to adapt a fundamentally open and experimental approach to disciplines, research questions as well as their potential for addressing societal issues. Therefore, 'interdisciplinarity' not only aims at crossing the boundaries of disciplines, but is also a challenge for research governance, funding agencies and policy makers. It stresses the necessity of investing in high risk- and curiosity-driven research initiatives as key drivers for research advancement as well as the developing of new practices and criteria for evaluating research projects and results. However, gaps persist between the widespread rhetoric for support of 'interdisciplinarity' and the realities of practice within the realm of research itself, as well as the institutional environments in which the research should be carried out. Universities as a rule (with a few exceptions) have not implemented systematic reforms for lowering institutional barriers and creating favourable cultures for both research and education. ${ }^{4}$ With respect to this, experiment and risk often may be controversial principles in those environments that provoke constraints of a political and ideological nature, thus hampering the development of interdisciplinarity. The freedom to follow curiosity-driven research questions, whether those related to the Grand Challenges or the ones we can see on the horizon, is not met with the same enthusiasm everywhere inside and outside the scientific community.

What also has to be recognized is the fact that a particular concentrated effort has to be made to bridge the divide between the hard sciences, on the one hand, and the Social Sciences and the Humanities (SSH) on the other. This is crucial if we are to address the many-layered problems inherent in interdisciplinary research questions and challenges. Thus, the necessity of raising the level of awareness to bridge gaps and work across boundaries is very much felt throughout the entire research community. However, in turn, this underpins crucial necessities such as mutual understanding, trust and respect across the entire research spectrum, especially between the hard sciences and SSH.

An important outcome of the conference was the concrete recommendations sent to the High Level Group on maximizing the impact of EU Research and Innovation Programmes (popularly known as the Lamy Group). Two major points were especially stressed. These were considered to be a direct contribution to the ongoing work of the Lamy Group at the time. 
First and foremost, the eminent researchers and scholars present stressed over and over again the importance of multi- and transdisciplinarity in the triggering of 'innovation' in the broadest sense of the word. The Lamy Group was asked to stimulate the various EU research agencies as well as national contact points to highlight the criticality of multi- and transdisciplinarity as a trigger for innovation.

The second point came primarily out of the third session entitled 'Interdisciplinarity and academic career path' and it stressed the need to facilitate real opportunities for early-career researchers to meet and develop practical, problemrelated links, especially between scholars coming from very different research domains. There have been several excellent smaller scale initiatives that may be examples of best practice that could be developed in the future.

In view of the need for wide transdisciplinary collaboration, including high-risk areas, it was recommended that the Lamy Group should seek to further strengthen the role of the COST networking mechanisms, as a focus for European trans- and interdisciplinary linkages and training, especially in supporting early stage researchers, widening participation agendas and providing a low-cost 'test-lab' for an emerging field of unrealized potential.

The eight papers in this volume address many of the challenges mentioned above, but as stated at the very beginning, we see this contribution not only as part of an ongoing debate, but also as providing concrete recommendations for furthering 'interdisciplinarity'. In turn, this means addressing major challenges that humans face in a rapidly changing physical environment as well as all the societal risks that seem to be more prominent than ever. How to deal with this multitude of questions is not just a theoretical endeavour, but as Van Rensselaer Potter ${ }^{5}$ claims:

Wisdom may be defined as the knowledge of how to use knowledge for the social good. [...] Humanistic biologists should be organized into interdisciplinary scientific research and development groups with SURVIVAL as their first goal. Societal competence may be defined as a function of wisdom and knowledge.

\section{References}

1. C.P. Snow (1998) The Two Cultures (with Introduction by Stefan Collini) (Cambridge: Cambridge University Press).

2. J. Mittelstraß (1982) Wissenschaft als Lebensform. Reden über philosophischer Orientierungen in Wissenschaft und Universität (Frankfurt am Main: Suhrkamp Verlag).

3. H. Nowotny, P. Scott and M. Gibbons (2003) 'Mode 2' revisited. The new production of knowledge. Minerva, 41, pp. 179-194.

4. J. Thompson Klein (2010) Creating Interdisciplinary Campus Cultures: A Model for Strength and Sustainability (San Francisco, CA: Jossey-Bass/Association of American Colleges and Universities).

5. V.R. Potter (1971) Bioethics: Bridge to the Future (Englewood Cliffs, NJ: PrenticeHall), p. 183. 


\section{About the Author}

Milena Žic Fuchs is a Full Professor at the University of Zagreb. In 2010 she was elected Fellow of the Croatian Academy of Arts and Sciences and in 2013 a member of Academia Europaea. She served as the Croatian Minister of Science and Technology from 1999 to 2000. From 2009 to 2012 she was Chair of the Standing Committee for the Humanities of the European Science Foundation. During her mandate she instigated policy incentives for Multidisciplinary Research and SSH and, on the basis of her contributions, she is a member of the Center for Excellence for Integrative Bioethics at the University of Zagreb. From 2008 she was a member of the ERC Advanced Grant Panel SH4 'The Human Mind and Its Complexity', which she chaired from 2014 to 2015. In 2016 she was elected Member of the High Level Group on maximizing the impact of EU Research \& Innovation Programmes. In 2018 she was appointed a member of the JRC (Joint Research Centre) High-Level Peer Group of the European Commission. 